\title{
Transmit antenna subset selection for high-rate MIMO-OFDM systems in the presence of nonlinear power amplifiers
}

\author{
Ngoc Phuc Le*, Farzad Safaei and Le Chung Tran
}

\begin{abstract}
The deployment of antenna subset selection on a per-subcarrier basis in MIMO-OFDM systems could improve the system performance and/or increase data rates. This paper investigates this technique for the MIMO-OFDM systems suffering nonlinear distortions due to high-power amplifiers. At first, some problems pertaining to the implementation of the conventional per-subcarrier antenna selection approach, including power imbalance across transmit antennas and noncausality of antenna selection criteria, are identified. Next, an optimal selection scheme is devised by means of linear optimization to overcome those drawbacks. This scheme optimally allocates data subcarriers under a constraint that all antennas have the same number of data symbols. The formulated optimization problem to realize the constrained scheme could be applied to the systems with an arbitrary number of multiplexed data streams and with different antenna selection criteria. Finally, a reduced complexity strategy that requires smaller feedback information and lower computational effort for solving the optimization problem is developed. The efficacy of the constrained antenna selection approach over the conventional selection approach is analyzed directly in nonlinear fading channels. Simulation results demonstrate that a significant improvement in terms of error performance could be achieved in the proposed system with a constrained selection compared to its counterpart.
\end{abstract}

Keywords: Antenna subset selection; MIMO-OFDM UWB systems; Nonlinear power amplifier; Power balancing; Linear optimization

\section{Introduction}

Recent years have seen a great demand for very fast data speeds in wireless multimedia applications. One of the most attractive techniques that could deliver high-rate transmission is multi-input multi-output orthogonal frequency division multiplexing (MIMO-OFDM) [1]. The major benefits of this technique resulting from OFDM include high spectral efficiency and robustness against intersymbol interference (ISI) in multipath fading channels [1]. Simultaneously, an increased capacity and/or diversity gain could be achieved with MIMO [2,3]. Among various MIMO schemes, antenna selection appears to be promising for OFDM wireless systems. This is mainly due to low-cost implementation and small amount of feedback information required, in comparison with other precoding methods [4]. In addition, this scheme is shown to

\footnotetext{
* Correspondence: pnl750@uow.edu.au

School of Electrical, Computer and Telecommunications Engineering,

University of Wollongong, Wollongong, New South Wales 2522, Australia
}

be effective in equivalent isotropic radiated power (EIRP)restricted systems, such as ultra-wideband (UWB) $[5,6]$.

Many research works have considered the application of antenna selection in OFDM systems, e.g., in [7-13]. In general, they can be categorized into two approaches: bulk selection (i.e., choosing the same antennas for all subcarriers) [7-10] and per-subcarrier selection (i.e., selecting antenna on each subcarrier basis) [10-13]. The main benefit of the latter over the former is that a much larger coding gain can be achieved by exploiting the frequency-selective nature of the channels [10]. Thus, per-subcarrier selection is very attractive for wideband communications. However, as the conventional persubcarrier selection method selects antennas independently for each subcarrier, a large number of data symbols may be allocated to some particular antennas. The input signal powers of the high-power amplifiers (HPAs) associated with these antennas might be very large, whereas those at the other antennas might be small. As a result, 
the HPAs on some antennas may operate in their inefficient power regions due to the small average powers of the input signals. Meanwhile, on the other antennas, nonlinear distortions, including in-band and out-of-band distortions, occur when the very large signal powers pass through the HPAs. The in-band distortion degrades error performance and system capacity [14], whereas the out-of-band distortion arising from the spectral broadening effect of the HPAs interferes the systems operating in the adjacent channels $[15,16]$.

It is obvious that the imbalance allocation of data subcarriers associated with the conventional per-subcarrier antenna selection scheme reduces the potential benefits of the antenna selection OFDM systems. One possible approach to deal with this problem is selecting transmit antennas under a constraint that the number of data subcarriers allocated to each antenna is equal. As a balance constraint is required, the constrained selection (i.e., power-balance selection) scheme should retain the benefits in terms of error performance or capacity as large as possible. Some research works have studied the constrained selection approach in the literature, such as [11-13]. In [11,12], allocation algorithms were developed to realize the constrained selection scheme. Meanwhile, the authors in [13] considered linear optimization to devise their constrained selection scheme. It was shown that the selection scheme based on optimization could offer a better performance than the suboptimal solutions in $[11,12]$. However, the formulated optimization problem in [13] is only applicable to OFDM systems where one antenna is active on each subcarrier. More importantly, to the best of our knowledge, all the existing works about constrained antenna selection, e.g., [11-13], only consider the effects of nonlinear HPAs on the system performance by means of simulations for demonstration purposes. This approach obviously has some limitations as it does not fully give an insight into the system characteristics. In particular, the question about whether antenna selection criteria originally derived in linear channels are still effective in nonlinear channels has not been addressed. This issue is of importance as the occurrence of nonlinear distortions may have impacts on the antenna selection criteria. Besides, the benefits in terms of error performance and/or capacity of the power-balance selection over the conventional scheme have not been analyzed directly for the systems suffering nonlinear distortions due to HPAs. It is clearly worth performing such an analysis, given that the efficacy of power-balance selection over its counterpart comes from the HPA nonlinearity. In addition, [11-13] only considered antenna selection schemes where data are transmitted from one antenna on each subcarrier. Thus, the achieved spectral efficiency was limited. To fulfill the expectation of delivering very fast data speeds for future wireless applications, antenna subset selection, where multiple data symbols are transmitted simultaneously from multiple antennas on each subcarrier, should be investigated.

In this paper, we propose and analyze constrained persubcarrier antenna subset selection for MIMO-OFDM systems in the presence of nonlinear distortions due to nonlinear HPAs. Moreover, we address the aforementioned limitations of the previous works. The major contributions of this paper could be summarized as follows:

1. A noncausal problem associated with the implementation of conventional per-subcarrier antenna selection in MIMO-OFDM systems suffering nonlinear distortions is identified for the first time. The noncausality arises because the impacts of nonlinear HPAs on transmitted data symbols need to be known in order to select a proper antenna subset for each subcarrier. Meanwhile, the calculations of these impacts require the total number of data subcarriers assigned on each antenna to be known.

2. An efficient constrained antenna subset selection scheme is proposed for MIMO-OFDM systems to overcome the drawbacks of the conventional scheme. The proposed scheme is realized based on a linear optimization problem that is formulated in systems with an arbitrary number of multiplexed data streams. Although the formulated optimization problem introduces an additional complexity in the proposed scheme, it can be solved efficiently by well-known linear programming methods.

3. A reduced complexity strategy that simultaneously requires a smaller number of feedback bits and lower computational effort to solve the optimization problem is proposed by exploiting the channel correlation between adjacent OFDM subcarriers.

4. The efficacy of the constrained antenna selection approach over the conventional approach is analyzed directly in the nonlinear fading channels. Specifically, we show that the average mean squared error (MSE) and the average signal-to-noise-plusdistortion ratio (SNDR) in the proposed system with a constrained selection are better than those in its counterpart. Numerical results are also provided to verify the analyses and demonstrate the improvement in terms of error performance in the proposed system.

The remainder of the paper is organized as follows. In Section 2, an antenna selection MIMO-OFDM system model with nonlinear HPAs is described. In Section 3, per-subcarrier antenna subset selection criterion is investigated in the systems suffering nonlinear distortions. In Section 4, an optimization problem for data subcarrier allocation with a power balancing is formulated. Performance analysis is carried out in Section 5. 
Simulation results are provided in Section 6. Finally, Section 7 concludes the paper.

\subsection{Notation}

Throughout this paper, a bold letter denotes a vector or a matrix, whereas an italic letter denotes a variable. (.)", $(.)^{T},(.)^{H},(.)^{-1}, \otimes, E\{$.$\} , and \operatorname{tr}\{$.$\} denote complex conjugation,$ transpose, Hermitian transpose, inverse, the Kronecker product, expectation, and the trace of a matrix, respectively. $\mathbf{I}_{n}$ indicates the $n \times n$ identity matrix, and $\mathbf{1}_{K}$ is a $K \times$ 1 vector of ones. $\operatorname{diag}(a)$ is the $n \times n$ diagonal matrix whose elements are the elements of vector $a$. $R$ indicates the set of real numbers.

\section{Antenna subset selection MIMO-OFDM systems with nonlinear HPAs}

\subsection{Transmitter}

We consider a MIMO-OFDM system with $K$ subcarriers, $n_{\mathrm{T}}$ transmit antennas, and $n_{\mathrm{R}}$ receive antennas as shown in Figure 1. At the transmitter, the input data are demultiplexed into $n_{\mathrm{D}}$ independent streams, where $n_{\mathrm{D}} \leq n_{\mathrm{T}}$ and $n_{\mathrm{D}} \leq n_{\mathrm{R}}$. Each data bit stream is then mapped onto $M$-ary phase shift keying (M-PSK) or $M$-ary quadrature amplitude modulation (M-QAM) constellation. Denote $q_{u}^{k}$ and $x_{i}^{k}, 1 \leq u \leq n_{\mathrm{D}}, 1 \leq i \leq n_{\mathrm{T}}, 0 \leq k \leq K-1$, to be the symbols that the subcarrier allocation block takes at its $u$ th input and outputs at its $i$ th output, respectively. The allocation block assigns the elements of $\mathbf{q}_{k}=\left[q_{1}^{k} q_{2}^{k} \ldots q_{n_{\mathrm{D}}}^{k}\right]^{T}$ to $n_{\mathrm{D}}$ selected antennas at the $k$ th subcarrier based on feedback information. As a result, only the $n_{\mathrm{D}}$ elements in a vector $\mathbf{x}_{k}=\left[x_{1}^{k} x_{2}^{k} \ldots x_{n_{\mathrm{T}}}^{k}\right]^{T}$ are assigned values from $\mathbf{q}_{k}$, whereas the others are zeros. Here, it is assumed that $E\left\{\mathbf{q}_{k} \mathbf{q}_{k}^{H}\right\}=$ $\sigma^{2} \mathbf{I}_{n_{\mathrm{D}}}$. The output sequences from the subcarrier allocation block are then fed into $K$-point inverse fast Fourier transform (IFFT) blocks. In this paper, the Nyquist sampling signal is considered. Thus, the discrete-time baseband OFDM signals can be expressed as

$$
s_{i}(n)=\frac{1}{\sqrt{K}} \sum_{k=0}^{K-1} x_{i}^{k} \mathrm{e}^{j 2 \pi n k / K}, 0 \leq n \leq K-1,1 \leq i \leq n_{\mathrm{T}} .
$$

Many power amplifier models, such as the Saleh model, SSPA model (or Rapp model), or soft envelope limiter (SEL) model, can be adopted in this system. For simplicity, we only consider the SEL model in this paper. Moreover, the SEL could model the state-of-the-art amplifier designs [15]. The $n$th output sample from the SEL is given by [17]

$$
\tilde{s}_{i}(n)= \begin{cases}s_{i}(n) & \text { if }\left|s_{i}(n)\right| \leq \sqrt{P_{\mathrm{o}, \text { sat }}} \\ \sqrt{P_{\mathrm{o}, \mathrm{sat}}} e^{<s_{i}(n)} & \text {,if }\left|s_{i}(n)\right|>\sqrt{P_{\mathrm{o}, \mathrm{sat}}}\end{cases}
$$

where $P_{\mathrm{o} \text {,sat }}$ is the output saturation power level of HPAs; $\left|s_{i}(n)\right|$ and $\angle s_{i}(n)$ denote the magnitude and phase of $s_{i}(n)$, respectively. Also, it is assumed that $P_{\mathrm{o} \text {,sat }}=P_{\mathrm{i} \text {,sat }}$, where $P_{\mathrm{i}, \text { sat }}$ is the input saturation power level.

For analytical tractability, we assume that the signals $s_{i}$ (n) are asymptotically independent and identically distributed (i.i.d.) Gaussian random variables. Note that this assumption, which is based on the central limit theorem [18], only holds when the number of data subcarriers on the $i$ th antenna, denoted as $K_{i}$, is large enough. By using Bussgang's theorem [19], the output of the nonlinear HPAs can be expressed as [17]

$$
\tilde{s}_{i}(n)=\alpha_{i} s_{i}(n)+\eta_{i}(n),
$$

where $\alpha_{i}$ is a scale factor, and $\eta_{i}(n)$ represents timedomain distortion noise that is uncorrelated with $s_{i}(n)$. The factor $\alpha_{i}$ and the variance $\sigma_{\eta_{i}}^{2}$ of $\eta_{i}(n)$ are, respectively, given by [17]

$$
\alpha_{i}=\frac{E\left\{s_{i}(n) \tilde{s}_{i}^{*}(n)\right\}}{E\left\{\left|s_{i}(n)\right|^{2}\right\}}=1-e^{-\vartheta_{i}^{2}}+\frac{\sqrt{\pi}}{2} \vartheta_{i} \operatorname{erfc}\left(\vartheta_{i}\right),
$$

and

$$
\sigma_{\eta_{i}}^{2}=E\left\{\left|\tilde{s}_{i}(n)\right|^{2}\right\}-\alpha_{i}^{2} E\left\{\left|s_{i}(n)\right|^{2}\right\}=\sigma_{K_{i}}^{2}\left[1-e^{-\vartheta_{i}^{2}}-\alpha_{i}^{2}\right],
$$

where $\sigma_{K_{i}}^{2}:=E\left\{\left|s_{i}(n)\right|^{2}\right\}=\sigma^{2} K_{i} / K$ is the average power of the input signal of the HPA on the $i$ th antenna, $\vartheta_{i}=\sqrt{P_{\mathrm{i}, \text { sat }} / \sigma_{K_{i}}^{2}}$ is the clipping ratio, and $\operatorname{erfc}(x)=\frac{2}{\sqrt{\pi}}$ $\int_{x}^{\infty} e^{-t^{2}} d t$ is a complementary error function. Note that $\sum_{i=1}^{n_{\mathrm{T}}} K_{i}=n_{\mathrm{D}} K$, thus $\sum_{i=1}^{n_{\mathrm{T}}} \sigma_{K_{i}}^{2}=n_{\mathrm{D}} \sigma^{2}$. In the system

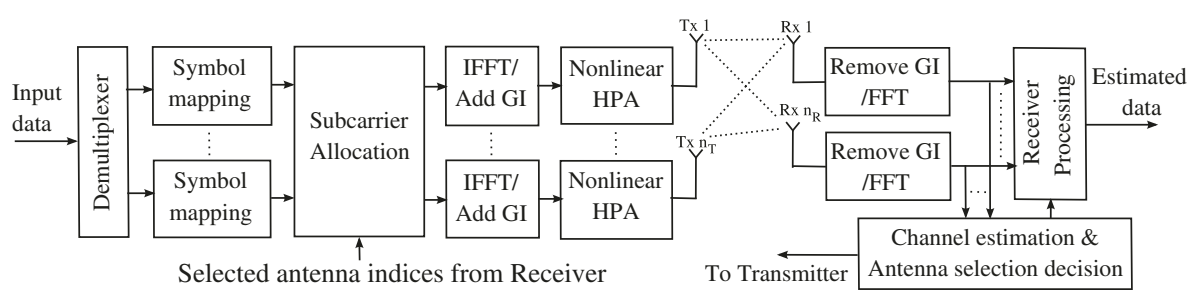

Figure 1 A simplified block diagram of a MIMO-OFDM system with per-subcarrier antenna subset selection. 
where the same number of data subcarriers is allocated to all antennas, we have $K_{i}=n_{\mathrm{D}} K / n_{\mathrm{T}}:=\bar{K}, \forall i=1,2, \ldots$, $n_{\mathrm{T}}$, and $\sigma_{K_{i}}^{2}=n_{\mathrm{D}} \sigma^{2} / n_{\mathrm{T}}:=\sigma_{\overline{\mathrm{K}}}^{2}, \forall i=1,2, \ldots, n_{\mathrm{T}}$. An input power back-off (IBO) of the HPAs is defined as $\mathrm{IBO}=P_{\mathrm{i}, \text { sat }} / \sigma_{K_{i}}^{2}$. Also, all HPAs are assumed to have the same nonlinear behavior. To illustrate the impacts of nonlinear distortions due to nonlinear HPAs on transmitted data symbols in the antenna selection OFDM system, we plot in Figure 2 the constellation diagrams of 16-QAM symbols in two scenarios: imbalance allocation and balance allocation of data subcarriers. It can be seen that although the nonlinear distortions is present in both scenarios, the data symbols in the scenario of the imbalance data-subcarrier allocation is more distorted than those in the other scenario. In other words, the level of nonlinear distortion is smallest when data subcarriers are equally allocated across transmit antennas.

\subsection{Receiver}

At the receiver, the received signal at each antenna is fed into the FFT block after the guard interval (GI) is removed. The system model in the frequency domain corresponding to the $k$ th subcarrier can be expressed as [20]

$$
\begin{aligned}
\mathbf{y}_{k} & =\mathbf{H}_{k} \mathbf{\alpha} \mathbf{x}_{k}+\mathbf{H}_{k} \mathbf{d}_{k}+\mathbf{n}_{k} \\
& =\underline{\mathbf{H}}_{k} \underline{\mathbf{\alpha}}_{k}+\underline{\mathbf{H}}_{k} \underline{\mathbf{d}}_{k}+\mathbf{n}_{k},
\end{aligned}
$$

where

$$
\mathbf{x}_{k}=\left[\begin{array}{llll}
x_{1}^{k} & x_{2}^{k} & \ldots & x_{n_{\mathrm{T}}}^{k}
\end{array}\right]^{T},
$$

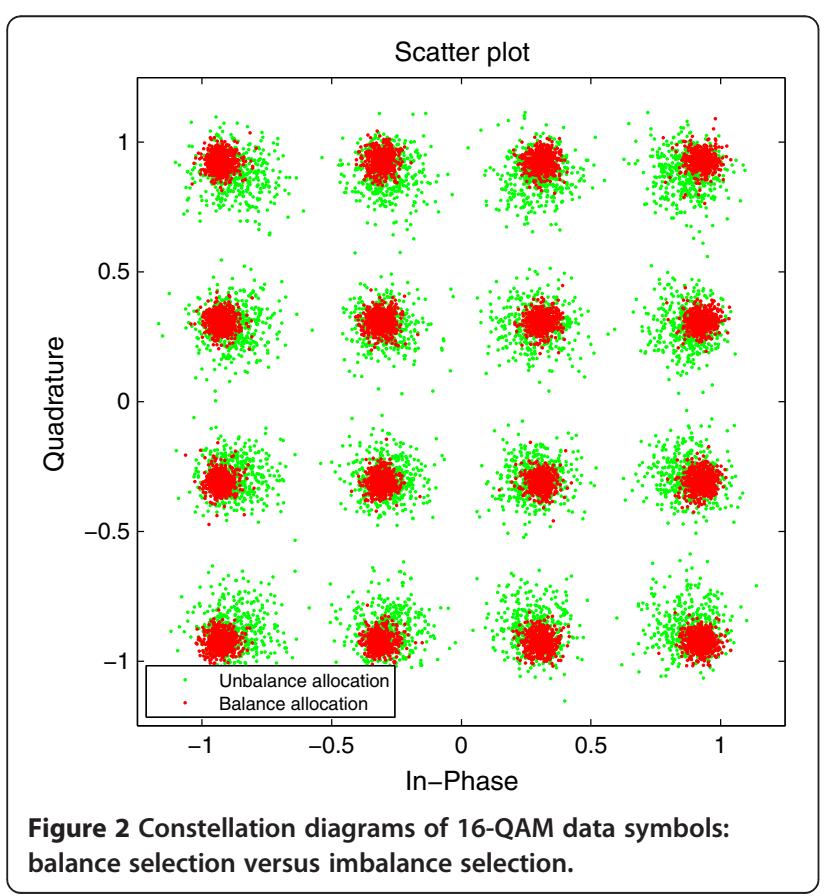

$$
\begin{aligned}
& \mathbf{H}_{k}=\left[\begin{array}{cccc}
h_{1,1}^{k} & h_{1,2}^{k} & \ldots & h_{1, n_{\mathrm{T}}}^{k} \\
h_{2,1}^{k} & h_{2,2}^{k} & \ldots & h_{2, n_{\mathrm{T}}}^{k} \\
\ldots & \ldots & \ldots & \ldots \\
h_{n_{\mathrm{R}}, 1}^{k} & h_{n_{\mathrm{R}}, 2}^{k} & \ldots & h_{n_{\mathrm{R}}, n_{\mathrm{T}}}^{k}
\end{array}\right], \\
& \mathbf{\alpha}=\operatorname{diag}\left(\left[\begin{array}{llll}
\alpha_{1} & \alpha_{2} & \ldots & \alpha_{n_{\mathrm{T}}}
\end{array}\right]\right), \\
& \mathbf{d}_{k}=\left[\begin{array}{llll}
d_{1}^{k} & d_{2}^{k} & \ldots & d_{n_{\mathrm{T}}}^{k}
\end{array}\right]^{T}, \\
& \mathbf{n}_{k}=\left[\begin{array}{llll}
n_{1}^{k} & n_{2}^{k} & \ldots & n_{n_{\mathrm{R}}}^{k}
\end{array}\right]^{T}, \\
& \mathbf{y}_{k}=\left[\begin{array}{llll}
y_{1}^{k} & y_{2}^{k} & \ldots & y_{n_{\mathrm{R}}}^{k}
\end{array}\right]^{T} .
\end{aligned}
$$

In the above equations, $h_{j, i}^{k}$ indicates the channel coefficient between the $i$ th transmit antenna and the $j$ th receive antenna. $d_{i}^{k}$ denotes the frequency-domain distortion noise at the $i$ th transmit antenna. Also, $y_{j}^{k}$ and $n_{j}^{k}$ denote the received signal and the thermal noise at the $j$ th receive antenna, respectively. The effective channel matrix $\underline{\mathbf{H}}_{k}$, the effective scale factor $\underline{\boldsymbol{\alpha}}=\operatorname{diag}\left(\left[\underline{\alpha}_{1} \underline{\alpha}_{2} \ldots \underline{\alpha}_{n_{\mathrm{D}}}\right]\right)$, and the effective distortion noise $\underline{\mathbf{d}}_{k}=\left[\underline{d}_{1}^{k} \underline{d}_{2}^{k} \ldots \underline{d}_{n_{\mathrm{D}}}^{k}\right]^{T}$ are obtained by eliminating the columns of $\mathbf{H}_{k}$, the rows of $\boldsymbol{\alpha}$, and the elements of $\mathbf{d}_{k}$ that correspond to the unselected transmit antennas, respectively. The distortion noise $d_{i}^{k}$ can be modeled as a zero-mean complex Gaussian random variable with variance $\sigma_{d_{i}}^{2}=\sigma_{\eta_{i}}^{2}$ (i.e., $\sigma_{d_{i}}^{2}$ is equal to that of the time-domain distortion noise). Note that as clipping is performed on the Nyquist-rate samples, all the subcarriers on the $i$ th antenna experience the same attenuation $\alpha_{i}$ and the variance $\sigma_{d_{i}}^{2}$ [17]. Thus, the factors of $\boldsymbol{\alpha}$ and $\underline{\boldsymbol{\alpha}}$, the variance of $\mathbf{d}$, denoted as $\boldsymbol{\sigma}_{d}^{2}=\operatorname{diag}$ $\left(\left[\sigma_{d_{1}}^{2} \sigma_{d_{2}}^{2} \ldots \sigma_{d_{n_{\mathrm{T}}}}^{2}\right]\right)$, and the variance of $\underline{\mathbf{d}}$, denoted as $\boldsymbol{\sigma}_{\underline{d}}^{2}=\operatorname{diag}\left(\left[\sigma_{\underline{d}_{1}}^{2} \sigma_{\underline{d}_{2}}^{2} \ldots \sigma_{\underline{d}_{n \mathrm{D}}}^{2}\right]\right)$, are the same for all subcarriers. Here, the indices $k$ associated with $\alpha_{i}$ and $\sigma_{d_{i}}^{2}$ are dropped for simplicity. The thermal noise is modeled as a Gaussian random variable with zero mean and $E\left\{\mathbf{n}_{k} \mathbf{n}_{k}^{H}\right\}=$ $\sigma_{n}^{2} \mathbf{I}_{n_{\mathrm{R}}}$. Also, it is assumed that per-subcarrier power loading is not an option due to the limited feedback rate and the strict regulation of a power spectral mask, such as in UWB systems.

Several MIMO detection techniques can be employed in this system to detect signals. For simplicity, we only consider a zero-forcing (ZF) receiver. Supposed that the perfect channel state information is available at the receiver, the equalized signal at the $k^{\text {th }}$ subcarrier is computed as [21]

$$
\tilde{\mathbf{q}}_{k}=\mathbf{G}_{k}^{+} \mathbf{y}_{k}=\mathbf{q}_{k}+\underline{\mathbf{\alpha}}^{-1} \underline{\mathbf{d}}_{k}+\mathbf{G}_{k}^{+} \mathbf{n}_{k},
$$

where $\mathbf{G}_{k}=\underline{\mathbf{H}}_{k} \underline{\mathbf{\alpha}}$, and $\mathbf{G}_{k}^{+}=\left(\mathbf{G}_{k}^{H} \mathbf{G}_{k}\right)^{-1} \mathbf{G}_{k}^{H}$ denotes the Moore-Penrose pseudo-inverse of a matrix $\mathbf{G}_{k}$. It can be 
seen from (13) that the estimated symbols consist of the desired component $\mathbf{q}_{k}$, the distortion noise after equalization $\underline{\mathbf{\alpha}}^{-1} \underline{\mathbf{d}}_{k}$, and the thermal noise after equalization $\mathbf{G}_{k}^{+} \mathbf{n}_{k}$. Note that to characterize the impacts of nonlinear distortions on the system performance, many other physical layer impairments, such as channel estimation error or I/Q imbalance, were not taken into consideration in this paper. For the case of existing errors in channel estimation, the readers are referred to [22], where the performance of a MIMO system in the presence of both nonlinear distortions and channel estimation errors is investigated. Although [22] does not consider antenna selection OFDM systems, the obtained results are useful for analyzing this system.

\section{Per-subcarrier antenna subset selection criteria in the presence of nonlinear HPAs}

\subsection{Per-subcarrier antenna subset selection criteria}

In a MIMO-OFDM system with conventional persubcarrier subset selection, antenna subsets are selected independently for each subcarrier. On each subcarrier, only $n_{\mathrm{D}}$ antennas out of $n_{\mathrm{T}}$ available transmit antennas are active. Denote $\Gamma_{\gamma} \gamma=1,2, \ldots \Gamma$, to be the $\gamma$ th subset consisting of $n_{\mathrm{D}}$ selected antennas, where $\Gamma=\left(\begin{array}{l}n_{\mathrm{D}} \\ n_{\mathrm{T}}\end{array}\right)=$ $\frac{n_{\mathrm{T}} !}{n_{\mathrm{D}} !\left(n_{\mathrm{T}}-n_{\mathrm{D}}\right) !}$ is the number of all possible $n_{\mathrm{D}}$-element subsets. Each subset consists of $n_{\mathrm{D}}$ transmit antenna indices that are chosen based on the feedback information from the receiver. For example, when $n_{\mathrm{T}}=4$ and $n_{\mathrm{D}}=2$, then $\Gamma=6$, and all possible subsets $\Gamma_{\gamma} \gamma=1,2, \ldots 6$, are defined in Table 1 . The choice of the best antenna subset depends on a particular antenna selection criterion.

Several antenna selection criteria that were originally derived from linear channels, such as minimum mean squared error (MMSE) [9], maximum capacity [23], or maximum signal-to-noise ratio (SNR) [23] can be extended to this system. For brevity, only the MMSE criterion is analyzed in this paper. The MMSE criterion selects the best antenna subset from the viewpoint of minimum mean squared error (i.e., minimizing the Euclidean distance between the estimated symbols and

\section{Table 1 Antenna subsets}

\begin{tabular}{ll}
\hline $\boldsymbol{\gamma}$ & $\boldsymbol{\Gamma}_{\boldsymbol{\gamma}}$ \\
\hline 1 & $\{1,2\}$ \\
2 & $\{1,3\}$ \\
3 & $\{1,4\}$ \\
4 & $\{2,3\}$ \\
5 & $\{2,4\}$ \\
6 & $\{3,4\}$ \\
\hline$n_{\mathrm{T}}=4, n_{\mathrm{D}}=2$, and $\Gamma=6$. &
\end{tabular}

the transmit symbols). Therefore, it also aims to minimize the error rate. When a ZF receiver is used, the error covariance matrix corresponding to the $k$ th subcarrier and the subset $\boldsymbol{\Gamma}_{\gamma}$ is computed as

$$
\begin{aligned}
\mathbf{M S E}_{\gamma}^{k} & =E\left\{\left(\tilde{\mathbf{q}}_{k}-\mathbf{q}_{k}\right)\left(\tilde{\mathbf{q}}_{k}-\mathbf{q}_{k}\right)^{H}\right\} \\
& =E\left\{\left(\underline{\mathbf{\alpha}}^{-1} \underline{\mathbf{d}}_{k}+\mathbf{G}_{k}^{+} \mathbf{n}_{k}\right)\left(\underline{\mathbf{\alpha}}^{-1} \underline{\mathbf{d}}_{k}+\mathbf{G}_{k}^{+} \mathbf{n}_{k}\right)^{H}\right\} \\
& =E\left\{\left(\underline{\mathbf{\alpha}}^{-1} \underline{\mathbf{d}}_{k}\right)\left(\underline{\mathbf{\alpha}}^{-1} \underline{\mathbf{d}}_{k}\right)^{H}\right\}+E\left\{\left(\mathbf{G}_{k}^{+} \mathbf{n}_{k}\right)\left(\mathbf{G}_{k}^{+} \mathbf{n}_{k}\right)^{H}\right\} \\
& =\boldsymbol{\sigma}_{\tilde{d}}^{2}+\sigma_{n}^{2}\left(\mathbf{G}_{k}^{H} \mathbf{G}_{k}\right)^{-1},
\end{aligned}
$$

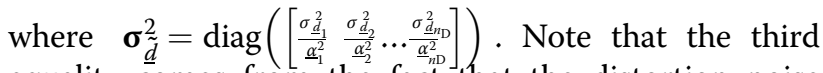
equality comes from the fact that the distortion noise and the thermal noise are independent. Recall that the MSE between the estimated symbols and the transmitted symbols is the trace of an error covariance matrix. Hence, the selected subset at the $k$ th subcarrier is determined by minimizing the trace of the MSE matrix, i.e.,

$$
\boldsymbol{\Gamma}_{\gamma}(k)=\arg \min _{\gamma=1, \ldots, \Gamma} \operatorname{tr}\left\{\mathbf{M S E}_{\gamma}^{k}\right\} .
$$

From (15), we draw two important remarks with respect to the deployment of per-subcarrier antenna selection in the MIMO-OFDM systems in the presence of nonlinear distortions:

1. If the same number of data subcarriers is allocated to all transmit antennas, the OFDM symbols in all antennas experience the same distortion characteristics (cf. (3) to (5)). Therefore, (15) can be simplified to

$$
\begin{aligned}
\Gamma_{\gamma}(k) & =\arg \min _{\gamma=1, \ldots, \Gamma} \sigma_{n}^{2} \operatorname{tr}\left\{\left(\mathbf{G}_{k}^{H} \mathbf{G}_{k}\right)^{-1}\right\} \\
& =\arg \min _{\gamma=1, \ldots, \Gamma} \operatorname{tr}\left\{\left(\underline{\mathbf{H}}_{k}^{H} \underline{\mathbf{H}}_{k}\right)^{-1}\right\},
\end{aligned}
$$

which is similar to that in the systems with ideal HPAs.

2. On the other hand, if the above condition is not satisfied, the per-subcarrier antenna selection criteria, e.g., MMSE criterion in (15), cannot be realized due to a noncausal problem. The noncausality arises because the selection of antenna subset for each subcarrier, i.e., calculating a metric $\mathrm{MSE}_{\gamma}^{k}$, requires the values $\underline{\alpha}$ and $\sigma_{\tilde{\tilde{d}}}^{2}$. Meanwhile, the calculations of these two values require the total number of data subcarriers assigned on each antenna to be known. To realize the per-subcarrier antenna selection, the criterion in (16) could be applied. However, as shown in (14) and (15), when the impacts of nonlinear HPAs are ignored, the selected antenna subset may not be the one that could obtain minimum MSE. Thus, the optimality of the selection criterion in terms of minimum MSE might not be fully achieved. 
Although only the MMSE criterion is considered in this paper, we note that the noncausal problem occurs with all per-subcarrier antenna selection criteria in the OFDM systems suffering nonlinear distortions.

\subsection{Feedback considerations}

With respect to a feedback mechanism used in this system, the selected antenna indices could be directly transmitted through reverse links in a time-division duplex (TDD) mode. In addition, it is typical in indoor wireless applications that the channel might not be changed during the transmission of several consecutive frames. In that scenario, the transmitter will reallocate data subcarriers according to the updated feedback information. Finally, in MIMO-OFDM systems with large values of $\Gamma$ and/or $K$, the number of feedback bits might be high. Reduced feedback could be realized by combining the subcarriers into a cluster and using only one antenna subset for all subcarriers in the cluster. This is due to the fact that neighboring subcarriers within each OFDM symbol are correlated. Therefore, it is likely that an optimal antenna subset for a particular subcarrier remains optimal for its neighbor subcarriers. If the cluster size is $L$, the number of feedback bits is reduced by $1 / L$. We propose the following criterion for choosing a proper subset for the $m$ th cluster, $1 \leq m \leq M, M=K / L$,

$$
\boldsymbol{\Gamma}_{\gamma}(m)=\arg \min _{\gamma=1, \ldots, \Gamma}\left\{\sum_{k=(m-1) L+1}^{m L} \operatorname{tr}\left\{\mathbf{M S E}_{\gamma}^{k}\right\}\right\}
$$

Note that the choice of value $L$ is a matter of tradeoff between feedback overhead and error performance. Moreover, the value $L$ is chosen based on the correlation characteristic of the channel frequency response. In MIMO-OFDM systems, the cross-correlation coefficients between two arbitrary subcarriers $k_{1}$ and $k_{2}$ can be expressed as [24]

$$
\begin{aligned}
\rho_{k_{1}-k_{2}}= & E\left\{\left[\mathbf{H}_{k_{1}}\right]_{i_{1}, j_{1}}\left[\mathbf{H}_{k_{2}}\right]_{i_{2}, j_{2}}^{*}\right\} \\
= & \sum_{t=0}^{T-1} \varphi_{t}^{2} e^{-j 2 \pi\left(k_{1}-k_{2}\right) t / K} \delta\left(i_{1}-i_{2}\right) \delta\left(j_{1}-j_{2}\right), \forall i_{1}, \\
& i_{2} \in 1, \ldots, n_{\mathrm{R}} ; \forall j_{1}, j_{2} \in 1, \ldots, n_{\mathrm{T}},
\end{aligned}
$$

where $\left[\mathbf{H}_{k}\right]_{i, j}$ denotes the $(i, j)$ th entry of the matrix $\mathbf{H}_{k}$, $\varphi_{t}$ (where $t=0,1, \ldots, T-1$ ) denotes the normalized channel power delay profile, i.e., $\sum_{t=0}^{T-1} \varphi_{t}^{2}=1$, and $\delta($.$) is$ the Kronecker-delta function. It can be seen from (18) that the frequency correlation coefficients depend on the difference between subcarriers $\left(k_{1}-k_{2}\right)$, rather than on the subcarriers themselves. Thus, given $\rho_{k_{1}-k_{2}}$, we can estimate $\left(k_{1}-k_{2}\right)$. In other words, the number of subcarriers in one cluster (i.e., the value of $L$ ) can be estimated, given the level of cross-correlation among the subcarriers within a cluster. The study of optimal designs regarding feedback reduction (e.g., deriving an optimal value of $L$ with respect to error performance-feedback rate tradeoff) is beyond the scope of this paper. The readers are referred to, e.g., $[25,26]$, for this topic of research.

\section{Optimization formulation for data subcarrier allocation with power balancing}

In Section 3, we have developed a per-subcarrier transmit antenna subset selection for the MIMO-OFDM system with nonlinear HPAs. As the conventional selection scheme selects the best antenna subset for each subcarrier, the number of data subcarriers assigned to each transmit antenna within one OFDM symbol period might be significantly different, depending on the channel conditions. Hence, the average input power of HPAs might vary significantly between OFDM symbol periods as well as among antennas. When the input powers on some antennas are small, the power efficiencies of the corresponding HPAs are reduced. On the other hand, large input powers result in severe distortion of signal. In this case, power back-off is required. However, the back-off will degrade the system performance. In addition, the imbalance allocation of the data subcarriers on antennas can lead to noncausality as discussed in Section 3. It is intuitive that these problems can be avoided if the same number of data subcarriers is allocated to all transmit antennas, as illustrated in Figure 3. When a balance selection of data subcarriers is required, the designed selection scheme should retain the benefits in terms of capacity or error performance as large as possible. To this end, we formulate a linear optimization problem to realize such a scheme.

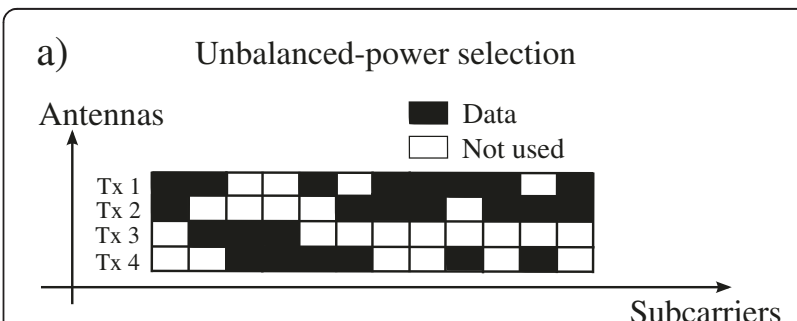

b) Balanced-power selection

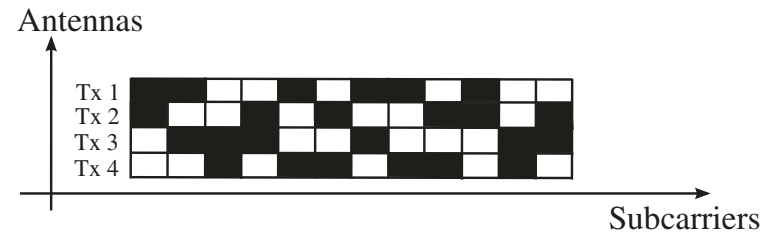

Figure 3 Illustration of per-subcarrier antenna subset selection $\left(n_{\mathrm{T}}=4, n_{\mathrm{D}}=2\right.$, and $\left.K=12\right)$. (a) Unbalance power selection.

(b) Balance power selection. 
As mentioned in the Introduction section, the linear optimization approach was considered for an OFDM system with $n_{\mathrm{D}}=1$ in [13]. Before proceeding to formulating a generalized optimization problem for systems with $n_{\mathrm{D}} \geq 1$, we make some evaluations with respect to the formulated problem in [13]:

1. A selection variable (i.e., optimization variable) in [13] was defined based on an antenna basis. When $n_{\mathrm{D}}>1$, a similar definition of a selection variable will result in binary nonlinear optimization problems. This is clearly not favorable from a practical viewpoint. As shown later in this section, binary linear optimization could be obtained by defining a selection variable based on a subset basis.

2. Only a system with full feedback was considered in [13]. In OFDM systems with large number of subcarriers, not only a large amount of feedback information is required but also the complexity to solve the optimization problem increases. Thus, it is of interest to formulate linear optimization working in conjunction with feedback reduction.

In the following, linear optimization problems are formulated for both full feedback and reduced feedback systems with an arbitrary number of data streams $n_{\mathrm{D}} \geq 1$.

\subsection{Optimization formulation}

We define a variable $z_{\gamma}^{k}$, where $z_{\gamma}^{k}=1$ if $\boldsymbol{\Gamma}_{\gamma}$ is chosen for the $k$ th subcarrier, and $z_{\gamma}^{k}=0$ otherwise. Also, denote $c_{\gamma}^{k}$ to be the cost associated with the chosen subset $\boldsymbol{\Gamma}_{\gamma}$. The type of the cost depends on the antenna selection criteria, e.g., $c_{\gamma}^{k}=\operatorname{tr}\left\{\mathbf{M S E}_{\gamma}^{k}\right\}$ if the MMSE selection criterion is used. The total cost function can be expressed as

$$
f=\sum_{k=0}^{K-1} \sum_{\gamma=1}^{\Gamma} c_{\gamma}^{k} z_{\gamma}^{k}
$$

As mentioned in Section 3, only the $n_{\mathrm{D}}$ antennas in this system are allowed to transmit data symbols on each subcarrier. This is equivalent to choosing only one subset of $n_{\mathrm{D}}$ elements among the $\Gamma$ subsets $\Gamma_{\gamma}, \gamma=1,2, \ldots \Gamma$, per subcarrier. Thus, the first constraint can be expressed as

$$
\sum_{\gamma=1}^{\Gamma} z_{\gamma}^{k}=1, \forall k=0,1, \ldots, K-1
$$

The second constraint is that all transmit antennas have the same number of allocated data subcarriers. In case when $K n_{\mathrm{D}}$ is not divisible by $n_{\mathrm{T}}$, some antennas will be allowed to have one more subcarrier than others. This will guarantee that the transmit power will be evenly distributed over the transmit antennas as much as it could. This constraint can be expressed as

$$
\sum_{k=0}^{K-1} z_{\gamma}^{k}=\lambda_{\gamma}, \gamma=1,2, \ldots, \Gamma,
$$

where the parameter $\lambda_{\gamma}$ is the number of times that the subset $\boldsymbol{\Gamma}_{\gamma}$ is selected. The values $\lambda_{\gamma}$ are chosen to satisfy

$$
\sum_{\psi \in \Psi^{i}} \lambda_{\psi} \leq\left\lceil\frac{n_{\mathrm{D}} K}{n_{\mathrm{T}}}\right\rceil, i=1,2, \ldots, n_{\mathrm{T}},
$$

where $\Psi^{i}$ denotes a set consisting of $\left(\begin{array}{l}n_{\mathrm{D}}-1 \\ n_{T}-1\end{array}\right)$ subsets $\boldsymbol{\Gamma}_{\gamma}$ which contains the $i$ th antenna, and $\lceil a\rceil$ indicates the smallest integer that is larger than or equal to $a$. For example, from Table 1, we have $\boldsymbol{\Psi}^{1}=\left\{\boldsymbol{\Gamma}_{1}, \boldsymbol{\Gamma}_{2}, \boldsymbol{\Gamma}_{3}\right\}, \boldsymbol{\Psi}^{2}=\left\{\boldsymbol{\Gamma}_{1}\right.$, $\left.\boldsymbol{\Gamma}_{4}, \boldsymbol{\Gamma}_{5}\right\}, \Psi^{3}=\left\{\boldsymbol{\Gamma}_{2}, \boldsymbol{\Gamma}_{4}, \boldsymbol{\Gamma}_{6}\right\}$, and $\boldsymbol{\Psi}^{4}=\left\{\boldsymbol{\Gamma}_{3}, \boldsymbol{\Gamma}_{5}, \boldsymbol{\Gamma}_{6}\right\}$. Note that if $K$ is divisible by $\Gamma$, (22) can be simplified to

$$
\lambda_{\gamma}=\frac{K}{\Gamma}, \forall \gamma=1,2, \ldots, \Gamma
$$

For instance, if $n_{\mathrm{T}}=4, n_{\mathrm{D}}=2$, and $K=12$, then $\lambda_{\gamma}=\frac{12}{6}=$ $2, \forall \gamma=1,2, \ldots, 6$. As all subsets are chosen twice, from Table 1, we know that each antenna has six data symbols (cf. Figure 3b).

The optimization problem is now a minimization of the cost function (19) subject to two constraints, (20) and (21). Note that in the system without power balancing, a problem of subcarrier allocation is equivalent to minimizing (19), subject to the constraint (20) only.

In what follows, we will represent the above optimization problem in a matrix form. Let us define vectors $\mathbf{z}=$ $\left(z_{1}^{0} \ldots z_{\Gamma}^{0} z_{1}^{1} \ldots z_{\Gamma}^{1} \ldots z_{1}^{K-1} \ldots z_{\Gamma}^{K-1}\right)^{T} \in\{0,1\}^{K T \times 1}$, and $\mathbf{c}=\left(c_{1}^{0} \ldots\right.$ $\left.c_{\Gamma}^{0} c_{1}^{1} \ldots c_{\Gamma}^{1} \ldots c_{1}^{K-1} \ldots c_{\Gamma}^{K-1}\right)^{T} \in \mathfrak{R}^{\mathrm{K} \Gamma \times 1}$. Then, (19) can be rewritten as $f=\mathbf{c}^{T} \mathbf{z}$. Also, the first and the second constraints can now be expressed as

$$
\mathbf{A}_{1} \mathbf{z}=\mathbf{1}_{K}
$$

where $\mathbf{A}_{1}=\mathbf{I}_{K} \otimes \mathbf{1}_{\Gamma}^{T} \in\{0,1\}^{K \times K \Gamma}$, and

$$
\mathbf{A}_{2} \mathbf{z}=\boldsymbol{\lambda}
$$

where $\mathbf{A}_{2}=\mathbf{1}_{K}^{T} \otimes \mathbf{I}_{\Gamma} \in\{0,1\}^{\Gamma \times K T}$ and $\boldsymbol{\lambda}=\left(\lambda_{1} \lambda_{2} \ldots \lambda_{\Gamma}\right)^{T} \in$ $\mathfrak{R}^{\Gamma \times 1}$. These constraints could be combined in a concise form as

$$
\mathbf{A z}=\mathbf{a}
$$

where $\mathbf{A}=\left(\mathbf{A}_{1}^{T} \mathbf{A}_{2}^{T}\right)^{T} \in\{0,1\}^{(K+\Gamma) \times K T}$ and $\mathbf{a}=\left(\mathbf{1}_{K}^{T} \boldsymbol{\lambda}^{T}\right)^{T} \in$ $\mathfrak{R}^{(K+\Gamma) \times 1}$. Consequently, the optimization problem becomes

$$
\begin{aligned}
& \underset{\mathbf{z} \in\{0,1\}^{K T \times 1}}{\min } \mathbf{c}^{T} \mathbf{z}, \\
& \text { subject to } \mathbf{A z}=\mathbf{a} .
\end{aligned}
$$


It is obvious that (27) has a canonical form of a binary linear optimization problem. Moreover, this binary optimization problem can be relaxed to a linear programming (LP) problem that has a solution $\mathbf{z} \in\{0,1\}^{K T \times 1}$ (see Appendix 1). As a result, the optimization problem in (27) can be solved efficiently by well-known linear programming methods, such as simplex methods or interior point methods [27]. When $n_{\mathrm{D}}=1$, the formulated problem in (27) is identical to the one in [13]. In addition, it is worth noting that as the optimization problem in (27) has been formulated in a way of minimizing the cost, a negative sign has to be included in the cost metric if capacity or SNR is used.

\subsection{Optimization in the system with reduced feedback}

In the system with feedback reduction, an efficient approach to formulate the optimization problem is based on a cluster basis rather than on a subcarrier basis. Let us define $z_{\gamma}^{m}$ and $c_{\gamma}^{m}=\sum_{k=(m-1) L+1}^{m L} \operatorname{tr}\left\{\mathbf{M S E}_{\gamma}^{k}\right\}$ to be the variable and the cost associated with the $m$ th cluster and the subset $\boldsymbol{\Gamma}_{\gamma}$ that is applied to all subcarriers within the $m$ th cluster, respectively. By doing similar steps as in Section 4.1, we arrive at an optimization formula similar to (27), except that

1. The number of variables is $\Gamma K / L$, i.e., $\mathrm{z} \in\{0,1\}^{(K T / L) \times 1}$.

2. A cost vector is $\mathrm{c} \in \mathfrak{R}^{(K \Gamma / L) \times 1}$ and its elements are $c_{\gamma}^{m}$.

3. Matrix A and vector a in the constraint will need to be modified accordingly.

With respect to the complexity of the proposed selection scheme, we note that the complexity to solve linear optimization using interior point methods can be reduced to $O\left(\left[(\Gamma K / L)^{3} / \ln (\Gamma K / L)\right] \zeta\right)$, where $O($.) denotes an order of complexity, and $\zeta$ is the bit size of the optimization problem [28]. Therefore, solving the optimization associated with reduced feedback (i.e., $L>1$ ) will require much lower computational effort compared to that on a subcarrier basis (i.e., $L=1$ ). As a result, the proposed system with this combined strategy could enjoy both small feedback overhead and low complexity for optimization.

\section{Performance analysis}

In Section 4, a linear optimization problem has been formulated to realize an optimal (constrained) selection scheme from a viewpoint of minimum MSE. In this section, we analyze the effectiveness of this selection scheme by showing that in the presence of nonlinear distortions, the average MSE, as well as the average SNDR, in the proposed system is better than that in the conventional system. Without loss of generality, it is assumed that all HPAs have the input saturation level of $P_{\mathrm{i}, \text { sat }}$ and operate with an input back-off of IBO $=P_{\mathrm{i}, \text { sat }} /$
$\sigma_{\bar{K}}^{2}$. In the conventional (unconstrained) system, the power back-off is required on the antennas where the numbers of the allocated data subcarriers are larger than $\bar{K}$, i.e., $K_{i}>\bar{K}$, to avoid error floor and other deleterious effects. This is equivalent to scaling the amplitudes of the signals on these antennas by a factor $\beta_{i}=\sqrt{\sigma \bar{K}^{2}} / \sigma_{K_{i}}^{2}$ $<1$. Meanwhile, the powers of the signals on the other antennas, i.e., $K_{i} \leq \bar{K}$, are not scaled up due to an EIRP restriction as well as the complexity of power loading.

Let us first rewrite the received signal $\mathbf{y}_{k}$ in (6) when the back-off operation is included as

$$
\begin{aligned}
\mathbf{y}_{k} & =\mathbf{H}_{k} \boldsymbol{\alpha} \boldsymbol{\beta} \mathbf{x}_{k}+\mathbf{H}_{k} \mathbf{d}_{k}+\mathbf{n}_{k} \\
& =\underline{\mathbf{H}}_{k} \underline{\boldsymbol{\alpha}} \underline{\boldsymbol{\beta}}_{\mathbf{q}_{k}}+\underline{\mathbf{H}}_{k} \underline{\mathbf{d}}_{k}+\mathbf{n}_{k},
\end{aligned}
$$

where $\boldsymbol{\beta}=\operatorname{diag}\left(\left[\beta_{1} \beta_{2} \ldots \beta_{n_{\mathrm{T}}}\right]\right)$, and $\underline{\boldsymbol{\beta}}=\operatorname{diag}\left(\left[\underline{\beta}_{1} \underline{\beta}_{2} \ldots \underline{\beta}_{n_{\mathrm{D}}}\right]\right)$ is obtained by eliminating the rows of $\beta$ that are corresponding to the unselected transmit antennas. Note that $\beta_{i}=1$ if no back-off is required on the $i$ th antenna. The error covariance matrix can now be expressed as (cf.(14))

$$
\begin{aligned}
\mathbf{M S E}^{k}= & E\left\{\left(\tilde{\mathbf{q}}_{k}-\mathbf{q}_{k}\right)\left(\tilde{\mathbf{q}}_{k}-\mathbf{q}_{k}\right)^{H}\right\} \\
= & E\left\{\left(\left(\underline{\boldsymbol{\alpha}} \underline{\boldsymbol{\beta}}^{-1} \underline{\mathbf{d}}_{k}\right)\left((\underline{\mathbf{\alpha}} \underline{\boldsymbol{\beta}})^{-1} \underline{\mathbf{d}}_{k}\right)^{H}\right\}\right. \\
& +E\left\{\left(\left(\underline{\mathbf{H}}_{k} \underline{\boldsymbol{\alpha}} \underline{\boldsymbol{\beta}}\right)^{+} \mathbf{n}_{k}\right)\left(\left(\underline{\mathbf{H}}_{k} \underline{\boldsymbol{\alpha}} \underline{\boldsymbol{\beta}}\right)^{+} \mathbf{n}_{k}\right)^{H}\right\} .
\end{aligned}
$$

From (29), we can express the MSE corresponding to the data symbol transmitted at the $u$ th selected antenna on the $k$ th subcarrier as

$$
\mathrm{MSE}^{k, u}=\frac{\sigma_{\underline{\underline{d}}_{u}}^{2}}{\underline{\alpha}_{u}^{2} \underline{\beta}_{u}^{2}}+\frac{\sigma_{n}^{2}}{\underline{\alpha}_{u}^{2} \underline{\beta}_{u}^{2}}\left[\left(\underline{\mathbf{H}}_{k}^{H} \underline{\mathbf{H}}_{k}\right)^{-1}\right]_{u, u},
$$

where $[\mathbf{A}]_{u, u}$ denotes the $(u, u)$ th entry of matrix $\mathbf{A}$. Thus, the average MSE across the subcarriers and transmit antennas can be calculated as

$$
\begin{aligned}
\overline{\mathrm{MSE}} & =\frac{1}{n_{\mathrm{D}} K} \sum_{k=0}^{K-1} \sum_{u=1}^{n_{\mathrm{D}}} \mathrm{MSE}^{k, u} \\
& =\frac{1}{n_{\mathrm{D}} K} \sum_{k=0}^{K-1} \sum_{u=1}^{n_{\mathrm{D}}} \frac{\sigma_{\underline{d}_{u}}^{2}+\sigma_{n}^{2}\left[\left(\underline{\mathbf{H}}_{k}^{H} \underline{\mathbf{H}}_{k}\right)^{-1}\right]_{u, u}}{\underline{\alpha}_{u}^{2} \underline{\beta}_{u}^{2}} .
\end{aligned}
$$

For notational simplicity, we denote

$$
F\left(u, \underline{\mathbf{H}}_{k}, K_{\Omega_{k}(u)}\right)=\frac{\sigma_{\underline{d}_{u}}^{2}+\sigma_{n}^{2}\left[\left(\underline{\mathbf{H}}_{k}^{H} \underline{\mathbf{H}}_{k}\right)^{-1}\right]_{u, u}}{\underline{\alpha}_{u}^{2}},
$$

where $\Omega_{k}$ is a mapping from the $u$ th selected antenna index to the $i$ th real antenna index at the $k$ th subcarrier, 
i.e., $i=\Omega_{k}(u), 1 \leq u \leq n_{\mathrm{D}}, 1 \leq i \leq n_{\mathrm{T}}$, which depends on the selected subset. Note that $\underline{\beta}_{u}^{2}$ and $\beta_{\Omega_{k}(u)}^{2}$ are the same in this paper. We can rewrite (31) as

$$
\overline{\mathrm{MSE}}=\frac{1}{n_{\mathrm{D}} K} \sum_{k=0}^{K-1} \sum_{u=1}^{n_{\mathrm{D}}} \frac{F\left(u, \underline{\mathbf{H}}_{k}, K_{\Omega_{k}(u)}\right)}{\underline{\beta}_{u}^{2}} .
$$

As mentioned above, in the unconstrained systems, the powers of signals on the antennas that have a large number of data subcarriers will be scaled by a factor $\beta_{\Omega_{k}(u)}^{2}<1$. Therefore, the average MSE in this system can now be expressed as

$$
\begin{array}{r}
\overline{\operatorname{MSE}}_{\text {im_bal }}=\frac{1}{n_{\mathrm{D}} K} \sum_{k=0}^{K-1}\left\{\sum_{u: \Omega_{k}(u) \in \mathbf{V}} F\left(u, \underline{\mathbf{H}}_{k}, K_{\Omega_{k}(u)}\right)\right. \\
\left.+\sum_{u: \Omega_{k}(u) \in \overline{\mathbf{V}}} \frac{F\left(u, \underline{\mathbf{H}}_{k}, \bar{K}\right)}{\beta_{\Omega_{k}}^{2}(u)}\right\},
\end{array}
$$

where $\mathbf{V}$ denotes a set of antennas in which the number of allocated data subcarriers on these antennas are smaller than or equal to $\bar{K}$, and $\overline{\mathbf{V}}$ is a set of the remaining antennas.

In the constrained system, the same number of data subcarriers $\bar{K}$ is allocated to all antennas. Thus, all subcarriers will be scaled by the same factor $\underline{\underline{\alpha}}$ and distorted by the distortion noises with the same variance $\bar{\sigma}_{\underline{d}}^{2}$. Recall that, for a given $\bar{K}$, the values of $\bar{\alpha}$ and $\bar{\sigma}_{d}^{2}$ can be calculated using (4) and (5), respectively. In addition, it is important to note that the effective channel matrix on the $k$ th subcarrier in the constrained system, denoted as $\underline{\mathbf{H}}_{k}$, is generally different from the channel matrix $\underline{\mathbf{H}}_{k}$ obtained in the unconstrained system because the selected antenna subsets may be different. From (31), we can express the average MSE in this system as

$$
\overline{\operatorname{MSE}}_{\text {bal }}=\frac{1}{n_{\mathrm{D}} K} \sum_{k=0}^{K-1} \sum_{u=1}^{n_{\mathrm{D}}} \frac{\bar{\sigma}_{\underline{d}}^{2}+\sigma_{n}^{2}\left[\left(\overline{\mathbf{H}}_{k}^{H} \underline{\mathbf{H}}_{k}\right)^{-1}\right]_{u, u}}{\underline{\bar{\alpha}}^{2}} .
$$

On the other hand, let us define $\Delta$ to be the difference in the total cost between the constrained and unconstrained schemes, i.e., (cf. (16) and (19))

$$
\Delta=\sum_{k=0}^{K-1} \sum_{u=1}^{n_{\mathrm{D}}}\left[\left(\overline{\mathbf{H}}_{k}^{H} \underline{\mathbf{H}}_{k}\right)^{-1}\right]_{u, u}-\sum_{k=0}^{K-1} \sum_{u=1}^{n_{\mathrm{D}}}\left[\left(\underline{\mathbf{H}}_{k}^{H} \underline{\mathbf{H}}_{k}\right)^{-1}\right]_{u, u} .
$$

Note that the value $\Delta$ is positive due to the fact that the total cost in the constrained optimization (i.e., minimization problem) is always larger than that in its unconstrained counterpart. Substituting (36) into (35), we arrive at

$$
\begin{aligned}
\overline{\mathrm{MSE}}_{\text {bal }} & =\frac{1}{n_{\mathrm{D}} K}\left\{\sum_{k=0}^{K-1} \sum_{u=1}^{n_{\mathrm{D}}} \frac{\bar{\sigma}_{\underline{d}}^{2}+\sigma_{n}^{2}\left[\left(\underline{\underline{\mathbf{H}}}_{k}^{H} \underline{\mathbf{H}}_{k}\right)^{-1}\right]_{u, u}}{\underline{\bar{\alpha}}^{2}}+\frac{\sigma_{n}^{2}}{\underline{\bar{\alpha}}^{2}} \Delta\right\} \\
& =\frac{1}{n_{\mathrm{D}} K}\left\{\sum_{k=0}^{K-1} \sum_{u=1}^{n_{\mathrm{D}}} F\left(u, \underline{\mathbf{H}}_{k}, \bar{K}\right)+\frac{\sigma_{n}^{2}}{\underline{\bar{\alpha}}^{2}} \Delta\right\} .
\end{aligned}
$$

The difference in the average MSE between the unconstrained and the constrained systems can now be computed as

$$
\begin{aligned}
& \Theta= \overline{\mathrm{MSE}}_{\mathrm{imbba}}-\overline{\mathrm{MSE}}_{\mathrm{bal}} \\
&=\frac{1}{n_{\mathrm{D}} K}\left\{\sum_{k=0}^{K-1} \sum_{u: \Omega_{k}(u) \in \mathbf{V}} F\left(u, \underline{\mathbf{H}}_{k}, K_{\Omega_{k}(u)}\right)\right. \\
&+\sum_{k=0}^{K-1} \sum_{u: \Omega_{k}(u) \in \overline{\mathbf{V}}} \frac{F\left(u, \underline{\mathbf{H}}_{k}, \bar{K}\right)}{\beta_{\Omega_{k}(u)}^{2}} \\
&\left.\quad-\sum_{k=0}^{K-1} \sum_{u=1}^{n_{\mathrm{D}}} F\left(u, \underline{\mathbf{H}}_{k}, \bar{K}\right)-\frac{\sigma_{n}^{2}}{\underline{\bar{\alpha}}^{2}} \Delta\right\} \\
&=\frac{1}{n_{\mathrm{D}} K}\left\{\sum _ { k = 0 } ^ { K - 1 } \sum _ { u : \Omega _ { k } ( u ) \in \mathbf { V } } \left(F\left(u, \underline{\mathbf{H}}_{k}, K_{\Omega_{k}(u)}\right)\right.\right. \\
&\left.\quad-F\left(u, \underline{\mathbf{H}}_{k}, \bar{K}\right)\right)+\sum_{k=0}^{K-1} \sum_{u: \Omega_{k}(u) \in \overline{\mathbf{V}}}\left(\frac{F\left(u, \underline{\mathbf{H}}_{k}, \bar{K}\right)}{\beta_{\Omega_{k}(u)}^{2}}\right. \\
&\left.\left.\quad-F\left(u, \underline{\mathbf{H}}_{k}, \bar{K}\right)\right)-\frac{\sigma_{n}^{2}}{\underline{\bar{\alpha}}^{2}} \Delta\right\} \\
&=\frac{1}{n_{\mathrm{D}} K}\left(I_{\mathbf{V}}+I_{\overline{\mathbf{V}}}+I_{\Delta}\right),
\end{aligned}
$$

where

$$
\begin{aligned}
& I_{\mathbf{V}}=\sum_{k=0}^{K-1} \sum_{u: \Omega_{k}(u) \in \mathbf{V}}\left(F\left(u, \underline{\mathbf{H}}_{k}, K_{\Omega_{k}(u)}\right)-F\left(u, \underline{\mathbf{H}}_{k}, \bar{K}\right)\right), \\
& I_{\overline{\mathbf{V}}}=\sum_{k=0}^{K-1} \sum_{u: \Omega_{k}(u) \in \overline{\mathbf{V}}} F\left(u, \underline{\mathbf{H}}_{k}, \bar{K}\right)\left(\frac{1-\beta_{\Omega_{k}(u)}^{2}}{\beta_{\Omega_{k}(u)}^{2}}\right) \\
& I_{\Delta}=-\frac{\sigma_{n}^{2}}{\underline{\underline{\alpha}}^{2}} \Delta
\end{aligned}
$$

It can be seen from (38) that the change in the average MSE when implementing balanced allocation compared to the case of unbalanced allocation comes from $I_{\mathrm{V}}, I_{\overline{\mathrm{V}}}$, and $I_{\Delta}$, where

- $I_{\mathrm{V}}$ is a kind of MSE penalty that is associated with data subcarriers on the antennas where $K_{i}<\bar{K}$. It can be seen from (4) and (5) that when $K_{i}$ increases, 
$\alpha_{i}$ decreases and $\sigma_{\eta}^{2}$ increases. Thus, the value of the function $F\left(u, \underline{\mathrm{H}}_{k}, K_{i}\right)$, defined in (32), increases when $K_{i}$ increases. Consequently, the value of $I_{\mathrm{V}}$ in (39) is always negative (i.e., $I_{\mathrm{V}}<0$ ).

- $I_{\overline{\mathrm{V}}}$ is a MSE benefit that is associated with data subcarriers on the antennas where $K_{i}>\bar{K}, i=\Omega_{k}$ (u). As the scale factor $\beta_{i}^{2}<1$, it is clear that $I_{\overline{\mathrm{V}}}>0$. The more data subcarriers are allocated to some particular antennas, the smaller the value $\beta_{i}^{2}=\sigma \bar{K}^{2} /$ $\sigma_{K_{i}}^{2}=\bar{K} / K_{i}$ is required, and thus, $I_{\overline{\mathrm{V}}}$ becomes larger.

- $I_{\Delta}$ is a kind of MSE penalty that is incurred because the chosen effective channel matrices in the constrained system are different from the ones in the unconstrained system. Note that $I_{\Delta}<0$ because $\Delta>0$ as mentioned before.

It is important to note that for a given system with defined HPAs in terms of nonlinear characteristics, only $I_{\Delta}$ among the three components depends on the effective channel matrices $\underline{\mathbf{H}}_{k}, k=0,1, \ldots, K-1$. Therefore, while different balanced selection schemes introduce different changes in the average MSE, the difference in the average MSE indeed comes from the difference in $I_{\Delta}$. From this observation, it is clear that to make the value $\Theta$, the difference in the average MSE between the unconstrained and constrained systems, become as positive as possible, the constrained selection method should result in the cost penalty $\Delta$ as small as possible. We note that the formulated optimization in (27) could achieve the minimum possible value of the total cost. Hence, with the definition of $\Delta$ as shown in (36), it is expected that the proposed constrained selection scheme based on linear optimization will guarantee the minimum achievable value of $\Delta$. In addition, an upper bound of the expected value of the cost penalty is derived in Appendix 2. Based on the obtained bound, it is observed that for fixed values of $n_{\mathrm{T}}$ and $n_{\mathrm{D}}$, the cost penalty becomes smaller when the number of receive antennas $n_{R}$ increases.

It is now necessary to evaluate the value of $\Theta$. As it is too challenging to mathematically evaluate $\Theta$ from a statistical viewpoint due to the fact that all components $I_{\mathrm{V}}, I_{\overline{\mathrm{V}}}$, and $I_{\Delta}$ are complicated and are dependent random variables, we perform a numerical evaluation of (38) instead. Figure 4 plots the empirical cumulative distribution function (CDF) of $I_{\mathrm{V}}, \mathrm{CDF}$ of $I_{\Delta}$, complementary CDF (CCDF) of $I_{\bar{V}}$, and CCDF of $\Theta$. These statistical distributions are obtained in the system with $n_{\mathrm{T}}=4, n_{\mathrm{D}}=2, n_{\mathrm{R}}=2, K=128$, and $\mathrm{IBO}=8 \mathrm{~dB}$ and are averaged over 400 channel realizations. Details about the other simulation parameters are described in Section 6 . The numerical results confirm that $I_{\mathbf{V}}<0, I_{\bar{V}}>0$, and $I_{\Delta}<0$. Moreover, as shown in Figure $4 \mathrm{~d}$, the probability (a)

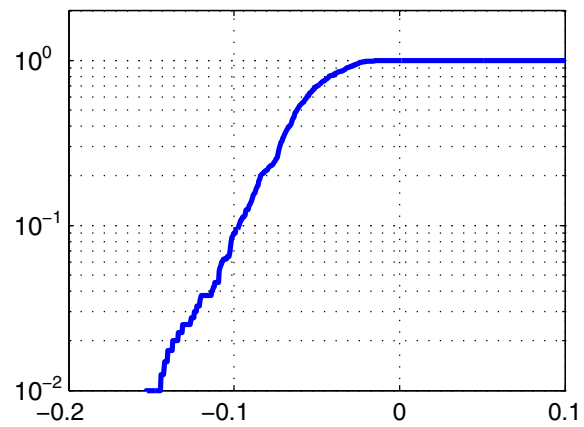

(c)

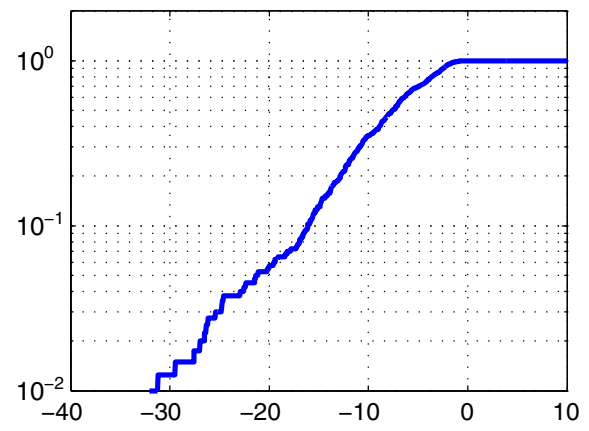

(b)

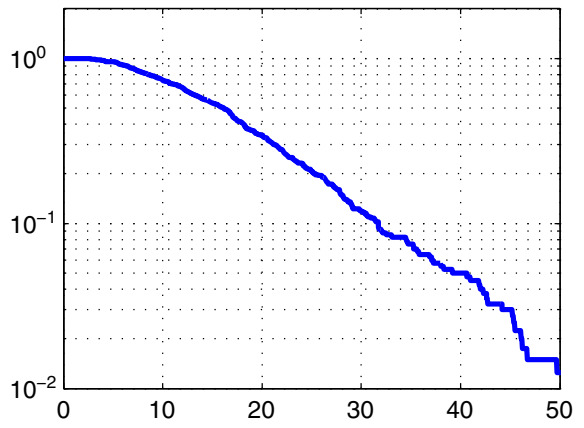

(d)

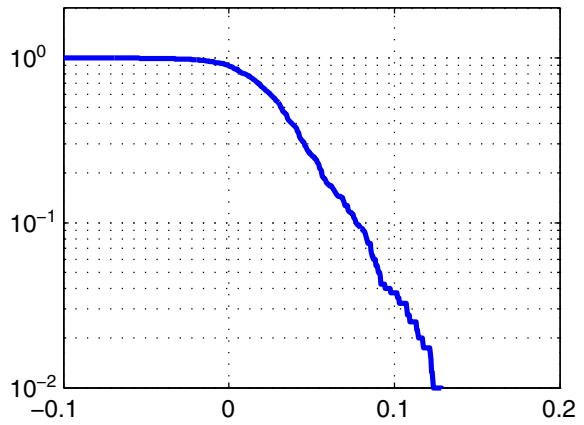

Figure 4 Statistical distributions. (a) CDF of $I_{v}$ (b) $C C D F$ of $I_{\bar{V}}$, (c) $\operatorname{CDF}$ of $I_{\Delta}$, and (d) $\operatorname{CCDF}$ of $\Theta$. 
of $\Theta$ being positive is very significant. Therefore, the proposed system could achieve a smaller average MSE (i.e., a better MSE performance) than that in the unconstrained system. In case that the receiver first estimates the value of $\Theta$ and then apply the constrained method only when $\Theta>$ 0 , then the value of $\Theta$ is always positive. In addition, in a spatial multiplexing MIMO system with a ZF receiver, we have $\mathrm{SNDR}^{k, u}=\sigma^{2} / \mathrm{MSE}^{k, u}$, where $\mathrm{SNDR}^{k, u}$ is the postprocessing SNDR corresponding to the data symbol transmitted at the $u$ th selected antenna on the $k$ th subcarrier [21]. Thus, it can be shown that the proposed system could achieve a better average SNDR than the unconstrained system. Error rate performance comparison will be provided and discussed in the next section.

The improvement in terms of MSE performance in the proposed system has been analyzed. It is also worth mentioning that the efficacy of the proposed system can be viewed from another perspective: peak-power reduction. In particular, we mathematically prove in [29] that the probability of occurrences of high peak power across antennas is smallest when the same number of data subcarriers is allocated to all the antennas. In other words, the proposed system can achieve peak-power reduction. With respect to a peak-to-average power ratio (PAPR) performance, an evaluation based on this metric depends on the specific definition of PAPR in the antenna selection OFDM setting, given that a PAPR metric is originally defined in single antenna OFDM systems. In case that the PAPR of the antenna selection OFDM system is defined as the maximum among all the PAPRs of the $n_{\mathrm{T}}$ transmit antennas, i.e.,

$$
\text { PAPR }_{\text {MIMO-OFDM }}=\max _{i=1,2, \ldots, n_{T}}\left\{\operatorname{PAPR}_{i}\right\},
$$

where $\mathrm{PAPR}_{i}$ is the PAPR on the $i$ th transmit antenna,

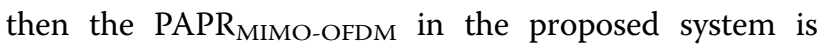
similar to that in the conventional system. This is because the CCDF of the $\mathrm{PAPR}_{i}$ on the $i$ th antenna, calculated as $\operatorname{Pr}\left(\mathrm{PAPR}_{i}>\mathrm{PAPR}_{0}\right)=1-\left(1-e^{-\mathrm{PAPR}_{0}}\right)^{K}$ [30], does not depend on the number of data subcarriers allocated on the $i$ th antenna. Thus, the CCDF of the PAPR MIMO-OFDM in both systems can be calculated as $[29,30]$

$$
\operatorname{Pr}\left(\mathrm{PAPR}_{\mathrm{MIMO}-\mathrm{OFDM}}>\mathrm{PAPR}_{0}\right)=1-\left(1-e^{-\mathrm{PAPR}_{0}}\right)^{n_{T} K} .
$$

In another case, if the PAPR of the system is defined as a ratio between the peak power across antennas and the average power across antennas, i.e.,

$$
\operatorname{PAPR}_{\text {MIMO-OFDM }}=\frac{n=0,1, \ldots, K-1 ; i=1,2, \ldots, n_{\mathrm{T}}\left\{\left|s_{i}(n)\right|^{2}\right\}}{\frac{1}{n_{\mathrm{T}} K} \sum_{n=0}^{K-1} \sum_{i=1}^{n_{\mathrm{T}}}\left|s_{i}(n)\right|^{2}},
$$

then the proposed system can achieve PAPR reduction. The reason is that while the average power across antennas is similar in both systems, i.e., $\left(1 / n_{\mathrm{T}} K\right) \sum_{n=0}^{K-1} \sum_{i=1}^{n_{\mathrm{T}}}\left|s_{i}(n)\right|^{2}=$ $n_{\mathrm{D}} \sigma^{2} / n_{\mathrm{T}}$, the proposed system can achieve the peak-power reduction as mentioned before. Note that all the analyses in this section hold for both full feedback and reduced feedback systems.

\section{Performance evaluations}

In this section, the error performance of the proposed system is evaluated via simulation results. The legacy WiMedia Multiband-OFDM UWB (MB-OFDM UWB) [31] is adopted for illustration. The simulation parameters are listed in Table 2. These parameters are based on a data-rate mode of $960 \mathrm{Mbps}$. Thus, the data rate in the proposed system when $n_{\mathrm{D}}=2$ is $1,920 \mathrm{Mbps}$. The system performance is measured in terms of packet error rate (PER) over the channel model of CM1 defined in the IEEE 802.15.3a channel model [32]. This channel is based on a measurement of a line-of-sight scenario where the distance between the transmitter and the receiver is up to $4 \mathrm{~m}$. Additionally, the multipath gains are modeled as independent log-normally distributed random variables. Perfect channel state information is assumed to be available at the receiver. Also, the feedback link is assumed to have no delay and is error-free. The average energy of transmitted data symbols is normalized to unity, i.e., $\sigma^{2}=1$.

Figure 5 compares the performance of the proposed system with that of the system without power balancing under different IBO values. It can be seen that there is a significant improvement in terms of PER performance in the proposed system. This agrees with the analysis in Section 5 that an imbalance allocation of data subcarriers in the unconstrained system results in a reduced average MSE, as well as a reduced average SNDR, compared to that in the proposed system. Similar observations can be made in the systems equipped with $n_{R}=3$ and $n_{\mathrm{R}}=4$ receive antennas as shown in Figure 6 .

\section{Table 2 Simulation parameters}

\begin{tabular}{ll}
\hline Parameter & Value \\
\hline Sampling frequency & $528 \mathrm{MHz}$ \\
FFT size & 128 \\
$\begin{array}{l}\text { Number of samples in } \\
\text { zero-padded suffix (ZPS) }\end{array}$ & 37 \\
Modulation scheme & Modified dual carrier modulation \\
(MDCM) & LDPC code defined in [31, Table 6.31], \\
Channel code & COde rate 3/4, decoder 10 iterations \\
IEEE 802.15.3a & CM1 \\
channel model & \\
\hline
\end{tabular}




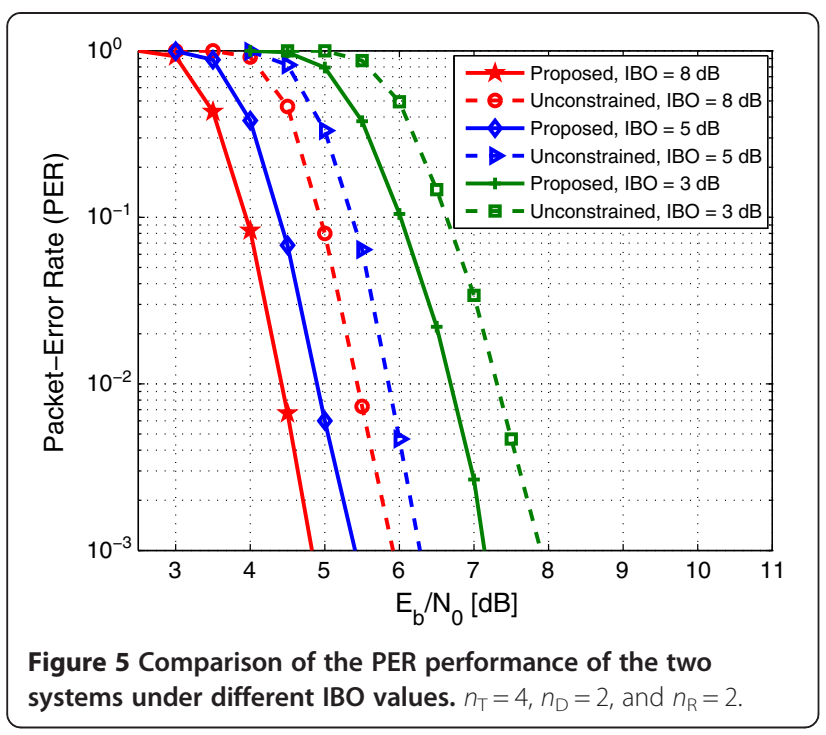

In Figure 7, we compare the PER performance of the proposed system under different antenna selection criteria, including MMSE, maximal SNR, and maximal capacity. It is clear that the MMSE criterion, which aims to minimize the Euclidean distance between the estimated symbols and the transmitted symbols, achieves a better error performance. Meanwhile, the capacity criterion, which aims to maximize the achievable rate, does not offer a good error performance, compared to the other criteria. This is due to the fact that the capacity criterion is based on a general capacity formula, which does not necessarily guarantee minimum error rate in the system with a ZF receiver [23].

Figure 8 shows the PER performance of the proposed system with the reduced complexity approach. Here, the

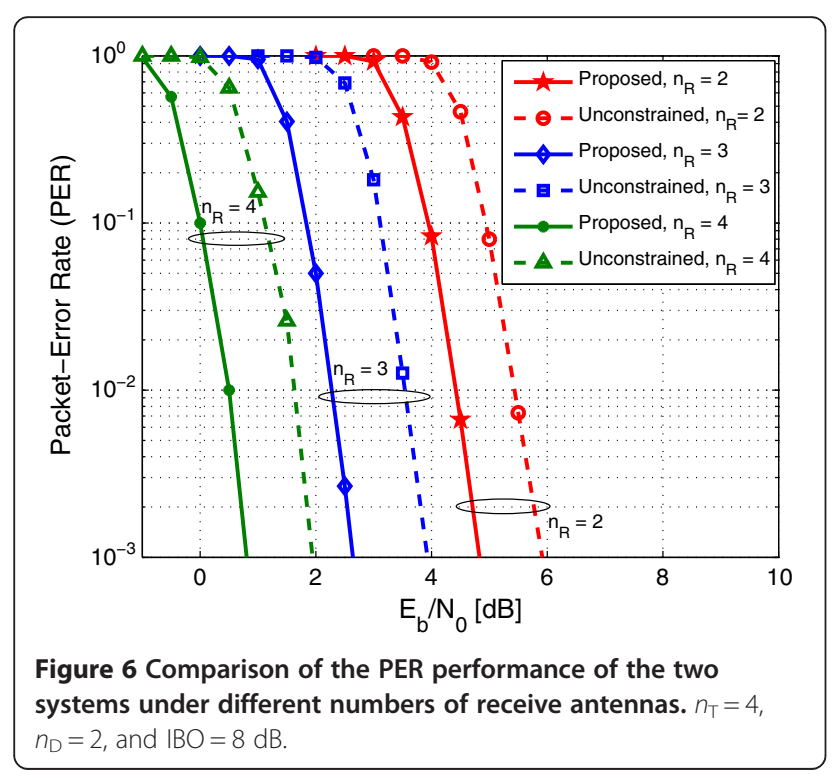

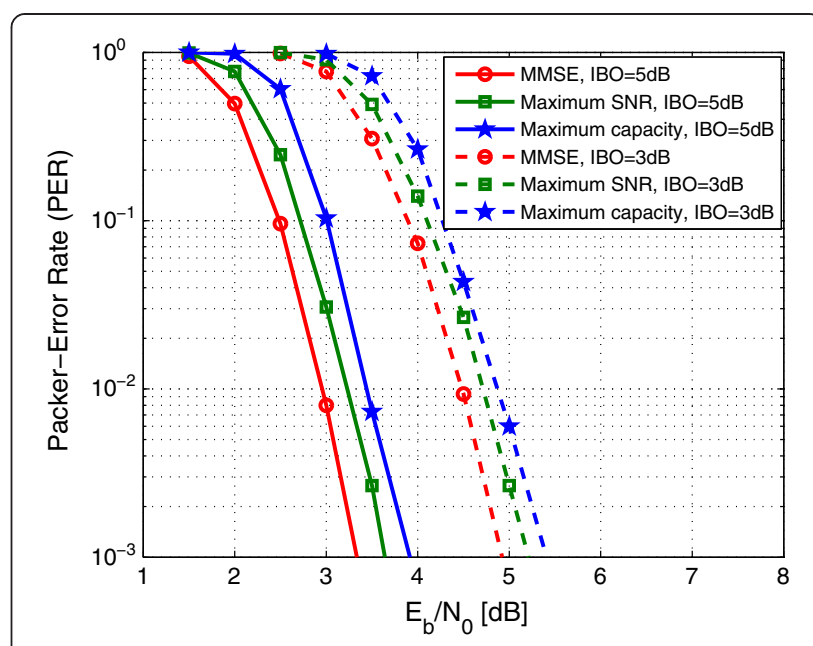

Figure 7 PER performance of the proposed system under different antenna selection criteria. $n_{T}=4, n_{D}=2$, and $n_{R}=3$.

feedback reduction of $L=8$ is used. As predicted, there is some loss in performance when applying feedback reduction compared to full feedback. However, we note that the system with feedback reduction requires only $12.5 \%$ of the number of feedback bits and has lower computational effort for solving the optimization problem. In addition, the proposed system with power balancing still outperforms its counterpart under reduced feedback. These results illustrate the efficacy of the proposed system with power balancing for practical MIMOOFDM wireless systems.

\section{Conclusions}

In this paper, a per-subcarrier antenna subset selection MIMO-OFDM system in the presence of nonlinear

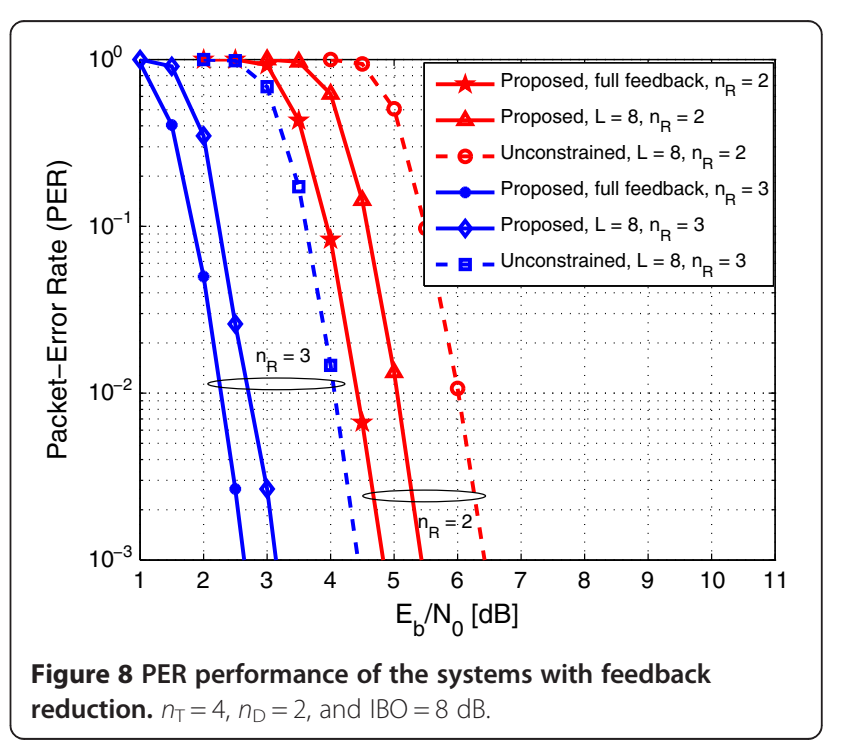


HPAs has been investigated. We have shown that the implementation of the conventional per-subcarrier selection in such a system suffers from the problem of performance degradation due to the large power back-off (resulting from an unequal allocation of data subcarriers across antennas) as well as the noncausality associated with the selection criteria. To overcome these drawbacks, we have proposed an optimal constrained selection scheme that can equally allocate data subcarriers among transmit antennas by means of linear optimization. The optimization problem to realize the proposed scheme is formulated in the system with an arbitrary number of multiplexed data streams. Moreover, it can be solved efficiently by existing methods. In addition, the reduced complexity strategy that requires less feedback information and lower computational effort for solving the optimization problem has been developed. We have analyzed the efficacy of the constrained antenna selection approach over the conventional approach directly in the nonlinear fading channels. The analysis could provide an insight into the system characteristics, i.e., the impacts of nonlinear HPAs on the performance of the antenna selection OFDM system. The simulation results show that a significant improvement in terms of error performance could be achieved in the system with a constrained antenna selection compared to its counterpart.

\section{Appendices}

\section{Appendix 1: linear relaxation of the binary optimization} in (27)

The optimization problem in (27) can be relaxed to linear programming (LP) relaxation using a similar approach as in [13] even though the constraint matrices in the two formulated problems are defined differently. Specifically, the feasible set of the LP relaxation of (27) can be expressed as

$$
\mathbf{S}=\left\{\mathbf{z} \in \mathfrak{R}^{K \Gamma \times 1} \mid \mathbf{A z}=\mathbf{a}, \mathbf{0}_{K T} \leq \mathbf{z} \leq \mathbf{1}_{K \Gamma}\right\},
$$

or

$$
\mathbf{S}=\left\{\mathbf{z} \in \Re^{K T \times 1} \mid \mathbf{B z} \leq \mathbf{b}\right\},
$$

where

$$
\begin{aligned}
\mathbf{B} & :=\left(\begin{array}{llll}
\mathbf{A}^{T} & -\mathbf{A}^{T} & \mathbf{I}_{K T} & -\mathbf{I}_{K T}
\end{array}\right)^{T}, \\
\mathbf{b} & :=\left(\begin{array}{llll}
\mathbf{a}^{T} & -\mathbf{a}^{T} & \mathbf{1}_{K T}^{T} & \mathbf{0}_{K T}^{T}
\end{array}\right)^{T} .
\end{aligned}
$$

As matrix A, defined in (26), is totally unimodular (i.e., every square submatrix of $\mathbf{A}$ has determinant $+1,-1$, or 0), it follows from [33] (also in [13, Proposition 1]) that $\mathbf{B}$ is also a totally unimodular matrix. On the other hand, vector b, defined in (48), is an integer vector. Therefore, the solution obtained by solving the LP relaxation using known programming methods is integral [33]. In other words, the optimal solution of the LP relaxation is also optimal for the original problem in (27).

\section{Appendix 2: upper bound of an expected value of cost penalty}

Let us first rewrite (36) as

$$
\Delta=\sum_{k=0}^{K-1} \operatorname{tr}\left\{\left(\underline{\mathbf{H}}_{k}^{H} \underline{\mathbf{H}}_{k}\right)^{-1}\right\}-\sum_{k=0}^{K-1} \operatorname{tr}\left\{\left(\underline{\mathbf{H}}_{k}^{H} \underline{\mathbf{H}}_{k}\right)^{-1}\right\}=\sum_{k=0}^{K-1} \Delta_{k},
$$

where

$$
\Delta_{k}=\operatorname{tr}\left\{\left(\underline{\overline{\mathbf{H}}}_{k}^{H} \underline{\overline{\mathbf{H}}}_{k}\right)^{-1}\right\}-\operatorname{tr}\left\{\left(\underline{\mathbf{H}}_{k}^{H} \underline{\mathbf{H}}_{k}\right)^{-1}\right\} .
$$

We now derive an upper bound of the expected value of $\Delta_{k}$. From (15), it can be seen that among all possible matrices $\underline{\mathbf{H}}_{k}$, the matrix $\underline{\mathbf{H}}_{k}$ with the lowest value of $\operatorname{tr}$ $\left\{\left(\underline{\mathbf{H}}_{k}^{H} \underline{\mathbf{H}}_{k}\right)^{-1}\right\}$ will be selected as the effective channel matrix for the $k$ th subcarrier in the unconstrained system. Meanwhile, the effective channel matrix associated with the $k$ th subcarrier in the constrained system is not necessarily the one with the lowest $\operatorname{tr}\left\{\left(\underline{\mathbf{H}}_{k}^{H} \underline{\mathbf{H}}_{k}\right)^{-1}\right\}$, i. e., $\operatorname{tr}\left\{\left(\underline{\overline{\mathbf{H}}}_{k}^{H} \underline{\overline{\mathbf{H}}}_{k}\right)^{-1}\right\} \geq \operatorname{tr}\left\{\left(\underline{\mathbf{H}}_{k}^{H} \underline{\mathbf{H}}_{k}\right)^{-1}\right\}$, due to the balance constraint. Hence, the expected value of $\Delta_{k}$ can be computed by using order statistics. In particular, an upper bound on the expected difference of two order statistics, the first and the $\gamma$ th, $1<\gamma \leq \Gamma$, is given by [34]

$$
\begin{aligned}
E\left\{\Delta_{k}\right\} & =E\left\{\operatorname{tr}\left\{\left(\underline{\overline{\mathbf{H}}}_{k}^{H} \underline{\mathbf{H}}_{k}\right)^{-1}\right\}\right\}-E\left\{\operatorname{tr}\left\{\left(\underline{\mathbf{H}}_{k}^{H} \underline{\mathbf{H}}_{k}\right)^{-1}\right\}\right\} \\
& \leq \sigma_{w} \sqrt{\frac{\Gamma(\Gamma-\gamma+2)}{\Gamma-\gamma+1}},
\end{aligned}
$$
where $\sigma_{w}^{2}$ is the variance of $\operatorname{tr}\left\{\left(\underline{\mathbf{H}}_{k}^{H} \underline{\mathbf{H}}_{k}\right)^{-1}\right\}$ that is as-
sumed to be the same for all matrices $\underline{\mathbf{H}}_{k}$.

On the other hand, suppose that the entries of the $n_{\mathrm{R}} \times n_{\mathrm{T}}$ matrix $\mathbf{H}_{k}$ are i.i.d. complex Gaussian random variables with zero mean and unit variance, then for any

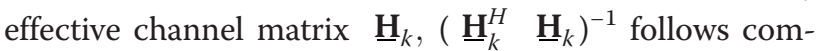
plex inverse Wishart distribution with $n_{\mathrm{R}}$ degrees of freedom [35]. When $n_{\mathrm{R}}>n_{\mathrm{D}}+1$, it is shown in [35, Lemma 6] that

$$
E\left\{\operatorname{tr}\left\{\left(\underline{\mathbf{H}}_{k}^{H} \underline{\mathbf{H}}_{k}\right)^{-1}\right\}\right\}=\frac{n_{\mathrm{D}}}{n_{\mathrm{R}}-n_{\mathrm{D}}}
$$


and

$$
\begin{aligned}
& E\left\{\left(\operatorname{tr}\left\{\left(\underline{\mathbf{H}}_{k}^{H} \underline{\mathbf{H}}_{k}\right)^{-1}\right\}\right)^{2}\right\} \\
& =\frac{n_{\mathrm{D}}}{n_{\mathrm{R}}-n_{\mathrm{D}}}\left(\frac{n_{\mathrm{R}}}{\left(n_{\mathrm{R}}-n_{\mathrm{D}}\right)^{2}-1}-\frac{n_{\mathrm{D}}-1}{n_{\mathrm{R}}-n_{\mathrm{D}}+1}\right) .
\end{aligned}
$$

Thus, the variance of $\operatorname{tr}\left\{\left(\underline{\mathbf{H}}_{k}^{H} \quad \underline{\mathbf{H}}_{k}\right)^{-1}\right\}$ can be computed as [18]

$$
\begin{aligned}
\sigma_{w}^{2} & =E\left\{\left(\operatorname{tr}\left\{\left(\underline{\mathbf{H}}_{k}^{H} \underline{\mathbf{H}}_{k}\right)^{-1}\right\}\right)^{2}\right\}-\left(E\left\{\operatorname{tr}\left\{\left(\underline{\mathbf{H}}_{k}^{H} \underline{\mathbf{H}}_{k}\right)^{-1}\right\}\right\}\right)^{2} \\
& =\frac{n_{\mathrm{D}} n_{\mathrm{R}}}{\left(n_{\mathrm{R}}-n_{\mathrm{D}}\right)^{2}\left[\left(n_{\mathrm{R}}-n_{\mathrm{D}}\right)^{2}-1\right]} .
\end{aligned}
$$

Substituting (54) into (51), we finally arrive at

$$
E\left\{\Delta_{k}\right\} \leq \sqrt{\frac{n_{\mathrm{D}} n_{\mathrm{R}}}{\left(n_{\mathrm{R}}-n_{\mathrm{D}}\right)^{2}\left[\left(n_{\mathrm{R}}-n_{\mathrm{D}}\right)^{2}-1\right]} \cdot \frac{\Gamma(\Gamma-\gamma+2)}{\Gamma-\gamma+1}} .
$$

\section{Competing interests}

The authors declare that they have no competing interests.

\section{Acknowledgements}

The authors would like to thank the anonymous reviewers for their helpful comments.

\section{Received: 9 September 2013 Accepted: 20 January 2014} Published: 13 February 2014

\section{References}

1. GL Stuber, JR Barry, SW McLaughlin, Y Li, MA Ingram, TG Pratt, Broadband MIMO-OFDM wireless communications. Proc. IEEE 92(2), 271-294 (2004)

2. GJ Foschini, Layered space-time architecture for wireless communication in a fading environment when using multi-element antennas. Bell. Syst. Tech. J. 1(2), 41-59 (1996)

3. V Tarokh, N Seshadri, AR Calderbank, Space-time codes for high data rate wireless communication: performance criterion and code construction. IEEE Trans. Inf. Theory 44(2), 744-765 (1998)

4. AF Molisch, MZ Win, MIMO systems with antenna selection. IEEE Microw. Mag. 5(1), 45-56 (2004)

5. S Roy, JR Foerster, VS Somayazulu, DG Leeper, Ultra wideband radio design: the promise of high-speed, short-range wireless connectivity. Proc. IEEE 92, 295-311 (2004)

6. CM Vithanage, JP Coon, SCJ Parker, On capacity-optimal precoding for multiple antenna systems subject to EIRP restrictions. IEEE Trans. Wireless Commun. 7(12), 5182-5187 (2008)

7. Z Tang, H Suzuki, IB Collings, Performance of antenna selection for MIMOOFDM systems based on measured indoor correlated frequency selective channels. Paper presented at the Australian telecommunications networks and applications conference (Melbourne, Australia, 2006), pp. 435-439

8. H Zhang, AF Molisch, J Zhang, Appling antenna selection in WLAN for achieving broadband multimedia communications. IEEE Trans. Broadcasting 52(4), 475-482 (2006)

9. Y Liu, Y Zhang, C Ji, WQ Malik, DJ Edwards, A low complexity receive antenna selection algorithm for MIMO-OFDM wireless systems. IEEE Trans. Vehicular Tech. 58(6), 2793-2802 (2009)

10. H Zhang, RU Nabar, Transmit antenna selection in MIMO-OFDM systems: bulk versus per-tone selection. Paper presented at the IEEE international conference on communications (Beijing, China, 2008), pp. 4371-4375

11. H Shi, M Katayama, T Yamazato, H Okada, A Ogawa, An adaptive antenna selection scheme for transmit diversity in OFDM systems. Paper presented at the IEEE vehicular technology conference (Atlantic City, NJ, USA, 2001), pp. 2168-2172

12. KH Park, YC Ko, MS Alouini, Low complexity transmit antenna selection with power balancing in OFDM systems. IEEE Trans. Wireless Commun. 9(10), 3018-3023 (2010)

13. M Sandell, J Coon, Per-subcarrier antenna selection with power constraints in OFDM systems. IEEE Trans. Wireless Commun. 8(2), 673-677 (2009)

14. R Prasad, OFDM for Wireless Communications Systems, 1st edn. (Artech House, Norwood, 2004)

15. I Ahmad, Al Sulyman, A Alsanie, A Alasmari, A Saleh, Spectral broadening effects of high power amplifiers in MIMO-OFDM relaying channels. EURASIP J. Wireless Commun. Netw. 2013, 32 (2013). doi:10.1186/1687-1499-2013-32

16. KG Grad, LE Larson, MB Steer, The impact of RF front-end characteristics on the spectral regrowth of communications signals. IEEE Trans. Microw. Theory 53(6), 2179-2186 (2005)

17. H Ochiai, $\mathrm{H}$ Imai, Performance of the deliberate clipping with adaptive symbol selection for strictly band-limited OFDM systems. IEEE J. Sel. Areas Commun. 18(11), 2270-2277 (2000)

18. JG Proakis, Digital Communications, 4th edn. (McGraw Hill Inc., New York, 2001)

19. JJ Bussgang, Cross-correlation functions of amplitude-distorted Gaussian signals, Technical Report 216 MIT (Research Laboratory Electronics, Cambridge, 1952)

20. P Banelli, Theoretical analysis and performance of OFDM signals in nonlinear fading channels. IEEE Trans. Wireless Commun. 2(2), 284-293 (2003)

21. A Paulraj, R Nabar, D Gore, Introduction to Space-Time Wireless Communications, 1st edn. (Cambridge University Press, Cambridge, 2003)

22. EKS Au, WH Mow, Effect of non-linearity on the performance of a MIMO zeroforcing receiver with channel estimation errors. Paper presented at the IEEE international conference on communications (Glasgow, UK, 2007), pp. 4150-4155

23. RW Heath, A Paulraj, S Sandhu, Antenna selection for spatial multiplexing systems with linear receivers. IEEE Commun. Lett. 5(4), 142-144 (2001)

24. MR McKay, PJ Smith, HA Suraweera, IB Collings, On the mutual information distribution of OFDM-based spatial multiplexing: exact variance and outage approximation. IEEE Trans. Inf. Theory 54(7), 3260-3278 (2008)

25. J Choi, B Mondal, RW Heath Jr, Interpolation based unitary precoding for special multiplexing MIMO-OFDM with limited feedback. IEEE Trans. Signal Processing 54(12), 4730-4740 (2006)

26. H Zhang, Y Li, V Stolpman, NV Waes, A reduced CSI feedback approach for precoded MIMO-OFDM systems. IEEE Trans. Wireless Commun. 6(1), 55-58 (2007)

27. R Fletcher, Practical Methods of Optimization, 2nd edn. (Willey, New York, 1987)

28. KM Anstreicher, Linear programming in $O\left(\left(n^{3} / n n\right) L\right)$ operations. SIAM J. Optim. 9(4), 803-812 (1999)

29. NP Le, LC Tran, F Safaei, Energy-efficiency analysis of per-subcarrier antenna selection with peak-power reduction in MIMO-OFDM wireless systems. Manuscript submitted to Int. J. Antennas Propag. under review

30. RFH Fischer, M Hoch, Peak-to-average power ratio reduction in MIMO OFDM. Paper presented at the IEEE international conference on communications (Glasgow, UK, 2007), pp. 762-767

31. A Batra, W Abbott, A Amanullah, R Aiello, A Anandakumar, N Askar, Y Azenkot, R Babu, J Balakrishnan, N Belk, F Berens, P Berg, R Bertschmann, D Birru, K Boehlke, L Bogod, PK Boon, C Brabenac, M Bravo-Escos, V Brethour, S ten Brink, N Carboni, B Carney, S Chari, J Cheah, F Chin, S Coffey, A Dabak, J Decuir, S Emami et al., Multiband OFDM physical layer specification, release 1.5 (WiMedia Alliance, San Ramon, 2009)

32. J Foerster (ed.), IEEE P802.15-02/490r1-SG3a: Channel Modeling Sub-Committee Final Report (IEEE, Piscataway, 2003)

33. D Bertsimas, R Weismantel, Optimization Over Integers, 2nd edn. (Dynamic Ideas, Belmont, 2005)

34. BC Arnold, RA Groeneveld, Bounds on expectations of linear systematic statistics based on dependent samples. Ann. Stat. 7(1), 220-223 (1979)

35. A Lozano, AM Tulino, S Verdu, Multiple-antenna capacity in the low powerregime. IEEE Trans. Inf. Theory 49(10), 2527-2544 (2003)

\section{doi:10.1186/1687-1499-2014-27}

Cite this article as: Le et al.: Transmit antenna subset selection for high-rate MIMO-OFDM systems in the presence of nonlinear power amplifiers. EURASIP Journal on Wireless Communications and Networking 2014 2014:27. 